\title{
Kernos
}

Revue internationale et pluridisciplinaire de religion grecque antique

$33 \mid 2020$

Varia

\section{Citizenship in Classical Athens}

\section{Vinciane Pirenne-Delforge}

\section{(2) OpenEdition \\ Journals}

\section{Édition électronique}

URL : https://journals.openedition.org/kernos/3563

DOI : 10.4000/kernos.3563

ISSN : 2034-7871

\section{Éditeur}

Centre international d'étude de la religion grecque antique

\section{Édition imprimée}

Date de publication : 31 décembre 2020

Pagination : 332-334

ISBN : 978-2-87562-264-8

ISSN : 0776-3824

\section{Référence électronique}

Vinciane Pirenne-Delforge, "Citizenship in Classical Athens », Kernos [En ligne], 33 | 2020, mis en ligne le 31 décembre 2020, consulté le 04 décembre 2022. URL : http://journals.openedition.org/kernos/ 3563 ; DOI : https://doi.org/10.4000/kernos.3563

Ce document a été généré automatiquement le 4 décembre 2022

Tous droits réservés 


\title{
Citizenship in Classical Athens
}

\author{
Vinciane Pirenne-Delforge
}

\section{RÉFÉRENCE}

Josine Bцок, Citizenship in Classical Athens, Cambridge, Cambridge University Press, 2017. 1 vol. $15,5 \times 23,5 \mathrm{~cm}$, xix+328 p. ISBN : 978-0-521-19145-6.

1 Qui n'a pas entendu dans ses cours d'histoire grecque que la citoyenneté de la polis était réservée aux hommes, au sens des «mâles adultes"? L'affaire a longtemps été considérée comme entendue : les femmes, exclues des fonctions politiques, n'étaient pas citoyennes. L'historiographie des $\mathrm{XIX}^{\mathrm{e}}$ et $\mathrm{xx}^{\mathrm{e}}$ siècles abonde d'un tel constat et l'influente entreprise de recherche menée par le Copenhagen Polis Centre a largement contribué à le souligner, notamment dans les travaux de son chef de file, Herman Mogens Hansen. Même Nicole Loraux, dans une perspective d'étude pourtant peu centrée sur l'institutionnel, associait la faible représentation de l'adjectif Athenaios au féminin au déclassement social des femmes au sein de la cité athénienne : Les Enfants d'Athéna étaient forcément des mâles.

2 C'est cette prétendue évidence que Josine Blok (J.B.) interroge depuis des années, dans des articles individuels comme au sein d'un programme collectif financé par le Fonds néerlandais de la recherche. Ce dernier a produit de très beaux fruits, tels l'ouvrage de Sara Wijma sur les métèques, celui de Saskia Peels sur le terme hosios et sa famille ${ }^{1}$, et une collaboration soutenue de l'A. avec l'excellent épigraphiste Stephen Lambert. L'ouvrage ici recensé vient couronner ce parcours en livrant une réflexion aussi approfondie que nuancée sur la notion de citoyenneté dans l'Athènes classique. Pour ce faire, elle interroge de front la vision institutionnelle de la citoyenneté qui réserve aux hommes charge politique (archē) et charge judiciaire (krisis), selon la définition du citoyen qu'Aristote livre dans la Politique (1275a 22-24). Une telle définition, lue au prisme des évolutions politiques des deux derniers siècles en Europe et aux États-Unis, a durablement marqué la vision moderne de la citoyenneté antique, reléguant aux marges du système les esclaves, les femmes, les enfants, les étrangers. Or, comme le 
montre J.B. en son premier chapitre («Rethinking Athenian Citizenship »), la focale se modifie dès que l'on quitte la réflexion théorique d'Aristote pour plonger dans le concret de la vie des Athéniens, notamment par le biais des plaidoyers judiciaires. Ainsi, quand l'Athénien Euxithéos doit se défendre d'une éradication de la liste des citoyens de son dème en 346/5, Démosthène ne lui fait pas dire qu'il a détenu une charge politique ou qu'il a siégé au tribunal pour appuyer sa revendication d'être citoyen. Les arguments invoqués sont une filiation adéquate (ascendance citoyenne du côté du père et de la mère) et une participation active aux hiera qui marquent cette même filiation (par exemple le fait de figurer dans la liste de candidats potentiels pour l'élection du prêtre d'Héraclès dans son dème). En bref, ce que nous appelons «la religion " apparaît comme une composante déterminante de la citoyenneté et, à ce titre, elle ouvre la voie à une inclusion des femmes dans la réflexion sur ce thème. Faire partie de la cité au titre de citoyen athénien, c'est avant tout metechein ta hiera kai ta hosia, " partager les hiera et les hosia », tant au sein de l'oikos qu'au niveau de la polis.

C'est dès lors à reprendre minutieusement l'étude des notions complexes qu'impliquent les termes hieros et hosios que J.B. réserve son deuxième chapitre intitulé « $\mathrm{A}$ Bond between Polis and Gods ». Un tel « pacte » est fondé sur la réciprocité à long terme entre les humains et leurs dieux, soutenue par la pratique du don sous toutes ses formes. Une telle réciprocité est essentielle au bien-être et à la continuité de la polis. De cette continuité, la filiation est un élément central auquel J.B. consacre son troisième chapitre ("The Value of Descent»). La construction de la communauté impose d'inscrire la parenté biologique dans un réseau social qui assure la transmission optimale des biens (matériels et immatériels) d'une génération à l'autre, conformément aux règles de ladite communauté et de ses attentes au sens large. La manière proprement athénienne d'élaborer la filiation, et donc la citoyenneté qui en découle, est concrète, ancrée dans la parenté et dans la famille, et non déterminée par des critères abstraits en termes de participation. C'est une "institution» ancrée dont J.B. retrace finement l'évolution aux périodes archaïque et classique, en posant une série d'intéressantes hypothèses sur la situation antérieure aux réformes de Clisthène. Ce dernier élargira le corps des citoyens, sans pour autant changer les principes de la filiation, mais en intensifiant l'engagement citoyen. Le changement majeur interviendra en 451/0 avec l'obligation d'être né d'un père et d'une mère "citoyens " pour l'être à son tour.

4 Jusqu'ici, c'est à élargir la définition étroite d'Aristote en termes institutionnels que le livre s'est attaché. Avec le quatrième chapitre ( $($ Citizens, Male and Female: Vocabulary »), c'est la dimension féminine de la citoyenneté qui se fait davantage jour, par le biais de l'étude du vocabulaire politēs/politis, astos/astēe, Athenaios/Athenaia. De l'étude attentive menée par J.B. sur l'usage de ces mots, il ressort notamment que l'emploi symétrique du vocabulaire masculin et féminin ne présente pas de différence essentielle dans la manière masculine et féminine de metechein ta hiera kai ta hosia. En revanche, en termes quantitatifs, les différences sont importantes et la "citoyenneté féminine " que traduit le vocabulaire en question n'apparaît que rarement dans la documentation: "Considering the large proportion of the extant evidence dealing with the political aspects of public life in ancient Greece, this scarcity is to be expected 》 (p. 184).

5 Le cinquième chapitre forme, selon moi, le point d'orgue du volume ( Participation: Public Roles and Institutions »). J.B. y convoque en pleine lumière les charges publiques occupées par des femmes athéniennes en rompant une lance contre la vision 
strictement politique de la citoyenneté. Une étude attentive des notions de timē/timai et d'archē/archai permet de poser le cadre des devoirs et des honneurs tels qu'ils se traduisent dans les diverses charges publiques. J.B. est ainsi amenée à nuancer la dichotomie stricte entre archai politiques et timai sacerdotales, dans la mesure où certains magistrats portent des insignes sacerdotaux, «reçoivent leur time du foyer public » (Aristote, Pol. 1322b 26-30), offrent des sacrifices et bénéficient à ce titre de parts d'honneur à l'instar des prêtres et prêtresses. Quant aux distinctions des devoirs de leur charge qu'impliquerait la différence de genre entre ces derniers, J.B. montre que les droits et devoirs des prêtres et des prêtresses sont globalement identiques, à l'exception sans doute de l'opportunité pour une prêtresse de rendre elle-même les comptes au terme de son office. Les honneurs publics rendus aux unes et aux autres mettent tous en exergue l'opération sacrificielle, centrale dans le "pacte» avec les dieux et assumée par les hommes comme par les femmes, quoi qu'ait pu écrire le regretté Marcel Detienne sur le genre en matière de sacrifice. Dans ce même chapitre, les développements sur le rôle des genē sont particulièrement éclairants. J.B. montre notamment que leur timēen matière cultuelle est un bien hérité qui doit avoir impliqué une forme d'" endogamie " en leur sein afin qu'une épiclère ne soit pas susceptible d'emmener son kleros hors du genos. Les genē avaient donc le pedigree de la loi de Périclès sur la double ascendance bien avant qu'elle ne soit promulguée. En outre, les modes de dévolution des charges publiques pourraient avoir également puisé à l'exemple de la dévolution des prêtrises au sein des genē, surtout quand ces charges comportaient d'importants devoirs religieux.

6 L'ouvrage se referme sur un dernier chapitre («Outlook: Athenians and Others ») où l'A. explore la situation des individus libres «naturalisés » athéniens, de même que celle des métèques. L'étude de la relation de ces groupes spécifiques aux timai de la cité s'accompagne d'une brève évocation des questions de citoyenneté hors d'Athènes, une brièveté qui atteste, si besoin en était, la nécessité de disposer d'une documentation riche pour appuyer l'analyse. On saura gré à Josine Blok d'avoir posé au dossier complexe de la citoyenneté athénienne d'autres questions que celles du «tout institutionnel» et du «tout politique». En effet, se limiter à interroger ainsi la documentation témoigne d'un beau cas de myopie: les textes ne cessent de répéter qu'être citoyen, c'est metechein ta hiera kai ta hosia. À refuser de le voir, on perd en chemin des composantes essentielles de la cité athéniennes: ses divinités et ses femmes. J.B. les a justement rappelées à la barre et, même si le détail de telle ou telle des démonstrations de l'ouvrage sera discuté car le thème reste polémique ${ }^{2}$, ces membres à part entière de la polis ne pourront plus être relégués à ses marges.

\section{NOTES}

1. S. WIJMA, Embracing the Immigrant: The Participation of Metics in Athenian Polis Religion (5th-4th Century BC), Stuttgart, 2014 ; S. PEELS, Hosios: A Semantic Study of Greek Piety, Leiden, 2016.

2. Voir, par exemple, P. FRÖHLICH, «La citoyenneté grecque entre Aristote et les Modernes », CGG 28 (2016), p. 91-136. 


\section{AUTEURS}

\section{VINCIANE PIRENNE-DELFORGE}

Collège de France

Université de Liège 